\title{
Special AT-rich sequence binding protein 1 regulates the multidrug resistance and invasion of human gastric cancer cells
}

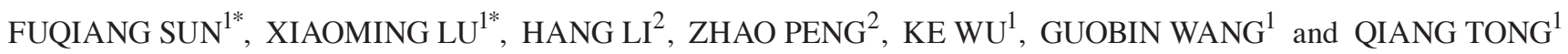 \\ Departments of ${ }^{1}$ Gastrointestinal Surgery and ${ }^{2}$ Laparoscopic Surgery, Union Hospital, Tongji Medical College, \\ Huazhong University of Science and Technology, Wuhan, Hubei 430022, P.R. China
}

Received January 21, 2012; Accepted April 2, 2012

DOI: $10.3892 / \mathrm{ol} .2012 .681$

\begin{abstract}
Special AT-rich sequence binding protein 1 (SATB1) is a nuclear factor that functions as a global chromatin organizer to regulate gene expression. Recent studies have suggested an oncogenic role of SATB1 in breast cancer. However, the role of SATB1 in gastric cancer, especially in regulating the malignant phenotypes, including multidrug resistance (MDR) and metastasis, remains poorly understood. In this study, the aggressive human gastric cancer cell line SGC7901 and its corresponding MDR variant SGC7901/VCR cells were used as a model. SATB1 expression was examined by RT-PCR and western blot analysis. Results showed that SATB1 was upregulated in SGC7901/VCR cells. An in vitro drug sensitivity assay demonstrated a positive correlation between SATB1 expression levels and drug resistance. Gain and loss of SATB1 function experiments further demonstrated that SATB1 contributes to MDR by inhibiting the accumulation of vincristine (VCR) in gastric cancer cells and protecting the cells from VCR-induced apoptosis. In addition, SATB1 may promote the invasion of gastric cancer cells. The present study provides a novel insight into the oncogenic role of SATB1 in gastric cancer, suggesting that SATB1 is a promising target for the therapy of drug-resistant and invasive gastric cancer.
\end{abstract}

\section{Introduction}

In the last decade, progress has been made in the treatment of patients with gastric cancer, which is one of the most prevalent malignancies worldwide. However, a significant percentage of gastric cancer patients fail to achieve complete

Correspondence to: Professor Qiang Tong, Department of Gastrointestinal Surgery, Union Hospital, Tongji Medical College, Huazhong University of Science and Technology, 1277 Jiefang Avenue, Wuhan, Hubei 430022, P.R. China E-mail: qiangtong@mail.hust.edu.cn; etongqiang@yahoo.com.cn

*Contributed equally

Keywords: gastric cancer, special AT-rich sequence binding protein 1 , multidrug resistance, invasion, chemotherapy remission, leading to a relapse or poor prognosis. Metastasis and multidrug resistance (MDR) have been regarded as major mechanisms underlying tumor relapse following successful surgical resections (1-3). Metastasis is the advanced stage of solid tumor progression and is a multi-step process that involves invasion, intravasation, extravasation to distant organs and finally growth of the secondary tumor. Chemotherapy is an important option in tumor treatment and contributes to the prevention of cancer metastasis and recurrence for surgical resection patients, but MDR is a significant clinical problem associated with chemotherapy $(4,5)$.

Chromatin architecture plays a significant role in the regulation of nuclear function and is crucial for the global changes in gene expression during tumor progression (6). Special AT-rich binding protein 1 (SATB1) is a well-characterized matrix attachment region (MAR)-binding protein. SATB1 organizes the chromatin into distinct loops via periodic anchoring of MARs to the nuclear matrix and functionally interacts with chromatin modifiers to suppress gene expression through histone deacetylation and nucleosome remodeling (7-10). SATB1 has been shown to directly regulate genes involved in cell proliferation, including c-myc $(11,12)$. Furthermore, previous studies have suggested that SATB1 is a master regulator of breast cancer growth and metastasis $(1,13)$. Notably, Li et al recently reported that SATB1 was able to upregulate certain genes associated with MDR and suppress drug-induced apoptosis in breast cancer, indicating the role of SATB1 in MDR (14).

Immunohistochemical assays revealed that the expression of SATB1 was higher in gastric cancer tissues than in normal gastric mucosa (15). Nevertheless, few studies have addressed the potential role of SATB1 in gastric cancer. Therefore, we investigated the role of SATB1 in gastric cancer with a particular focus on the biological characteristics of gastric cancer cells that are associated with MDR and metastasis.

\section{Materials and methods}

Cell culture. The human gastric adenocarcinoma cell line SGC-7901 (maintained in our laboratory) and its corresponding VCR-resistant variant SGC7901/VCR (obtained from the Xiangya Center Laboratory, Changsha, Hunan, China) were cultured in DMEM medium supplemented with $10 \%$ fetal bovine serum (FBS), $100 \mathrm{U} / \mathrm{ml}$ penicillin and $100 \mathrm{mg} / \mathrm{ml}$ streptomycin in a $37^{\circ} \mathrm{C}$ incubator with $100 \%$ humidity and 
$5 \% \mathrm{CO}_{2}$. In addition, to maintain the MDR phenotype, the drug-resistant cells were stimulated with $1 \mu \mathrm{g} / \mathrm{ml} \mathrm{VCR}$.

Plasmid construction and transfection. The coding region of SATB1 (GeneBank Accession number NM_002971) was amplified using the primers: forward: 5'-CCGACAACCACTACCTGA-3' and reverse: 5'-GTGGCACCTTCCAGGGTC-3', and subcloned into the mammalian expression vector pEX-M29 (GeneCopoeia, Rockville, MD, USA) to generate the plasmid pEX-SATB1. The recombinant construction was verified by DNA sequencing. pEX-SATB1 was prepared using the Fastfilter Endo-Free Plasmid Midi kit (Omega Bio-Tek, Norcross, GA, USA) according the manufacturer's instructions and transfected into SGC7901 or SGC7901/VCR cells using lipofectamine 2000 (Invitrogen, Carlsbad, CA, USA) according to the manufacturer's instructions. The cells were harvested after 36 or $48 \mathrm{~h}$ for the following experiments.

RNA interference. Three SATB1 siRNA oligonucleotides and one negative control sequence were designed based on the SATB1 sequence and synthesized by Guangzhou RiboBio Co., Ltd. (Guangzhou, China). SGC7901 cells were seeded at $2 \times 10^{5}$ cells/well in 6-well plates and cultured in serum-free medium. The following day, when the cells reached $70 \%$ confluence, $50 \mathrm{nM}$ siRNA was transfected into the cells using lipofectamine 2000 (Invitrogen) according to the manufacturer's instructions. After $6 \mathrm{~h}$, the cells were cultured in DMEM medium supplemented with $10 \%$ FBS. The cells were harvested at the indicated time points for the subsequent experiments.

$R T-P C R$ assay. Total RNA was isolated from the cultured cells using TRIzol reagent (Takara Bio, Inc., Shiga, Japan) according to the manufacturer's instructions. RT-PCR was performed using the StepOnePlus ${ }^{\mathrm{TM}}$ Real-Time PCR system (Applied Biosystems, Carlsbad, CA, USA). The primers used were: SATB1, forward: 5'-GTGGGTACGCGATCAACTGA-3' and reverse: 5'-TGTTAAAGCCAGTGCAA-3'; GAPDH (internal control), forward: 5'-TTGGTATCGTGGAAGGACTCA-3' and reverse: 5'-TGTCATCATATTTGGCAGGTT-3'. The PCR cycling conditions were denaturation at 48 cycles of $95^{\circ} \mathrm{C}$ for $30 \mathrm{sec}$, annealing at $52^{\circ} \mathrm{C}$ for $40 \mathrm{sec}$ and extension at $72^{\circ} \mathrm{C}$ for $40 \mathrm{sec}$. Finally, the PCR products were visualized on a $1.0 \%$ agarose gel containing $5 \mu \mathrm{g} / \mathrm{ml}$ ethidium bromide.

Western blotting. The cells were lysed with RIPA lysis buffer (Santa Cruz Biotechnology, Santa Cruz, CA, USA) and the protein concentration of the cell lysates was quantitated using the BCA method. Equal amounts of protein were loaded and separated via $10 \%$ SDS-PAGE, then transferred onto nitrocellulose membranes (Millipore, Billerica, MA, USA). The membranes were blocked with $5 \%$ non-fat milk for $2 \mathrm{~h}$ and then incubated with the SATB1 antibody (1:1,000 dilution; Epitomics, Burlingame, CA, USA) and $\beta$-actin antibody (1:2,000 dilution, Sigma, St. Louis, MO, USA) at $4^{\circ} \mathrm{C}$ overnight. Following three washes with TBST, the membranes were incubated with HRP-conjugated secondary antibodies (Boster, Wuhan, China) for $4 \mathrm{~h}$ at room temperature. The membranes were developed using an ECL kit (Santa Cruz Biotechnology) and exposed to $\mathrm{X}$-ray film. $\beta$-actin was used as a loading control.
In vitro invasion assay. The invasion behaviors of SGC7901 and its variant cells were detected using a Matrigel transmembrane invasion assay as described in a previous study (16). Briefly, $15 \mu \mathrm{l}$ of ice-cold Matrigel (Sigma) was spread over the transwell chambers ( $8-\mu \mathrm{m}$ pore size, Millipore) and incubated for $1 \mathrm{~h}$ at room temperature. Cells $\left(1 \times 10^{5}\right.$ cells/well $)$ were washed with PBS twice and then transferred to the upper chamber with $200 \mu \mathrm{l}$ serum-free medium. The lower chamber was filled with serum containing medium with the addition of fibronectin as the chemoattractant. The cells were allowed to invade across the Matrigel-coated membrane for $48 \mathrm{~h}$ at $37^{\circ} \mathrm{C}$ in $5 \% \mathrm{CO}_{2}$. The filter was removed gently and the cells on the upper surface were eliminated by wiping with a cotton swab. The cells that had invaded across the Matrigel and attached to the bottom were fixed and stained with hematoxylin and eosin (H\&E). The stained cells were counted under a microscope in 10 randomly selected high-power fields. The assay was performed for a minimum of three times independently.

In vitro drug sensitivity assay. The sensitivity of cells to VCR was evaluated using 3-(4,5-dimethylthiazol-2-yl)-2, 5-diphenyltetrazolium bromide (MTT) assay as described previously (17). Briefly, cells in the logarithmic phase were seeded in 96-well plates at a density of $1 \times 10^{4}$ cells/well. The following day, the cells were cultured in serum containing medium supplemented with a different concentration of VCR. Following 72 h, $50 \mu \mathrm{l}$ MTT (Sigma) was added into each well. After incubation for $4 \mathrm{~h}$, the supernatant was replaced with $150 \mu$ l dimethyl sulfoxide (Sigma). The optical density was read at a wavelength of $490 \mathrm{~nm}$ on a spectrophotometer (MPR-2100, Syntron, Carlsbad, CA, USA). The relative inhibitory rate on cell growth of different concentrations of VCR was calculated according to the following formula: $\mathrm{R}=\left(V_{I^{-}}-V_{2}\right) /$ $V_{2}$, where $\mathrm{R}$ equaled the relative inhibitory rate, $\mathrm{V}_{1}$ was the absorbance value of the control cells without VCR treatment and $\mathrm{V}_{2}$ was the absorbance value in the presence of VCR.

Flow cytometric analysis of apoptosis. The apoptotic rate was determined in SGC7901 and its variant cells using flow cytometry of propidium iodide (PI)-stained cells on a FACS Calibur flow cytometer (Becton Dickinson, San Jose, CA, USA). The cells were plated into 24-well plates and then treated with $1 \mu \mathrm{g} / \mathrm{ml} \mathrm{VCR} \mathrm{the} \mathrm{following} \mathrm{day.} \mathrm{After} \mathrm{two} \mathrm{days,}$ the pEZ-SATB1 plasmid or siRNA was tansfected into the cells. The cells were harvested, washed with PBS, fixed in $70 \%$ ethanol overnight at $4^{\circ} \mathrm{C}$ and stained with $50 \mathrm{mg} / \mathrm{ml}$ $\mathrm{PI}$ in the dark at room temperature for $30 \mathrm{~min}$. The sub- $\mathrm{G}_{1}$ peak was measured and analyzed using Cell Quest software (Becton Dickinson).

Intracellular VCR concentration analysis. Flow cytometry was used to detect the fluorescence intensity of intracellular VCR as described previously (18). Cells in the log phase were seeded in six-well plates at a density of $1 \times 10^{6}$ cells/well and cultured overnight at $37^{\circ} \mathrm{C}$. The cells were then incubated with $5 \mu \mathrm{g} / \mathrm{ml} \mathrm{VCR}$ for $1 \mathrm{~h}$. The cells were trypsinized and harvested for the detection of VCR accumulation, or alternatively cultured in RPMI-1640 for a further $30 \mathrm{~min}$ to detect the retention. Following washing twice with ice-cold PBS, the cells were analyzed by flow cytometry with an excitation 
A

SATB1

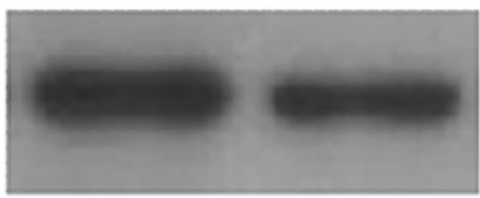

GAPDH

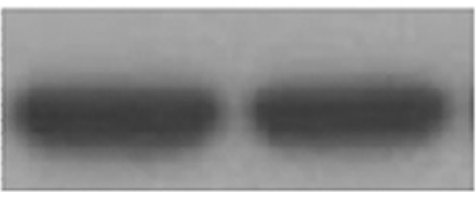

C

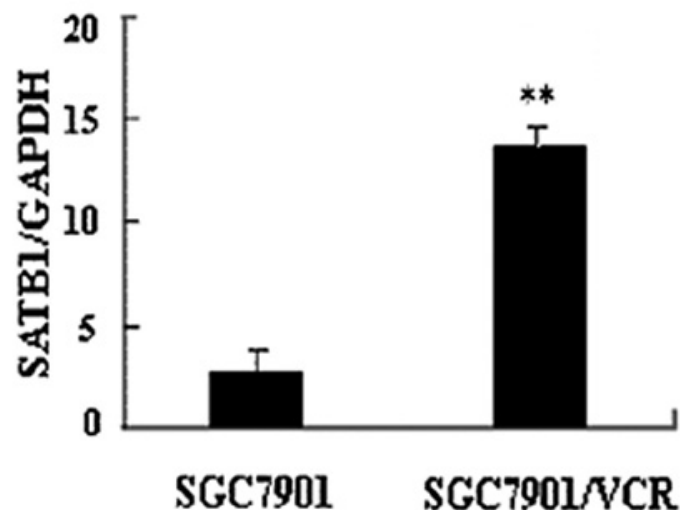

B

SGC7901/NCR SGC7901

SATB1

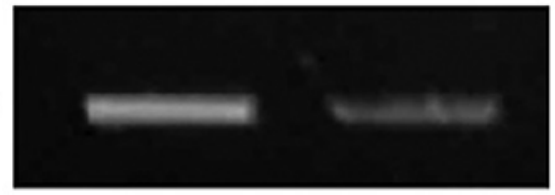

$\beta$-actin

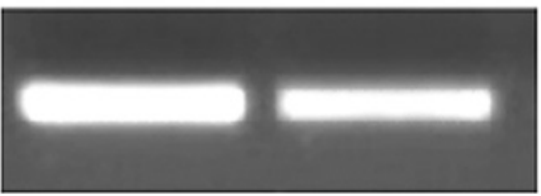

D

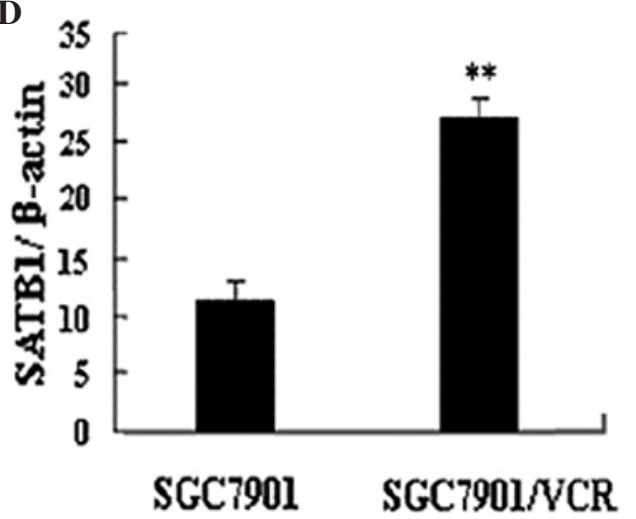

Figure 1. Overexpression of SATB1 in multidrug-resistant gastric cancer cells. (A) RT-PCR analysis revealed a higher SATB1 mRNA level in SGC7901/ VCR cells, compared with SGC7901 cells. GAPDH served as the internal control. (B) Western blots showed a higher SATB1 protein level in SGC7901/ VCR cells than in SGC7901 cells. $\beta$-actin served as the loading control. Representative images from three independent experiments with similar results. (C and D) Densitometric analysis of the results shown in (A) and (B), respectively. ${ }^{* *} \mathrm{P}<0.05$, SGC7901 cells vs. SGC7901/VCR cells. The data are shown as the mean \pm SD from three independent experiments. SATB1, special AT-rich sequence binding protein 1; VCR, vincristine.

wavelength of $488 \mathrm{~nm}$ and an emission of $575 \mathrm{~nm}$ to detect the intracellular VCR concentration.

Statistical analysis. Statistical analyses were performed using SPSS software (Chicago, IL, USA). Quantitative values are shown as the mean \pm SD. ANOVA and the Student's t-test were used to compare the differences. $\mathrm{P}<0.05$ was considered to indicate a statistically significant result.

\section{Results}

Expression of SATB1 is upregulated in MDR gastric cancer cells. This study compared SATB1 expression in VCR-resistant SGC7901/VCR cells and parent SGC7901 cells. RT-PCR and western blot analysis results revealed that the expression of SATB1 was higher in SGC7901/VCR cells than that in SGC7901 cells at the mRNA and protein levels (Fig. 1). These data suggest that SATB1 is associated with the development of MDR in gastric cancer cells.

SATB1 regulates $M D R$ in gastric cancer cells. To confirm that SATB1 is involved in the development of MDR in gastric cancer, stable gastric cancer cell lines were constructed in which SATB1 was overexpressed (SGC7901-SATB1 and SGC7901/ VCR-SATB1 cells) or depleted (SGC7901-siRNASATB1 and SGC7901/VCR-siRNASATB1 cells). RT-PCR revealed that the expression of SATB1 at the mRNA level was high,

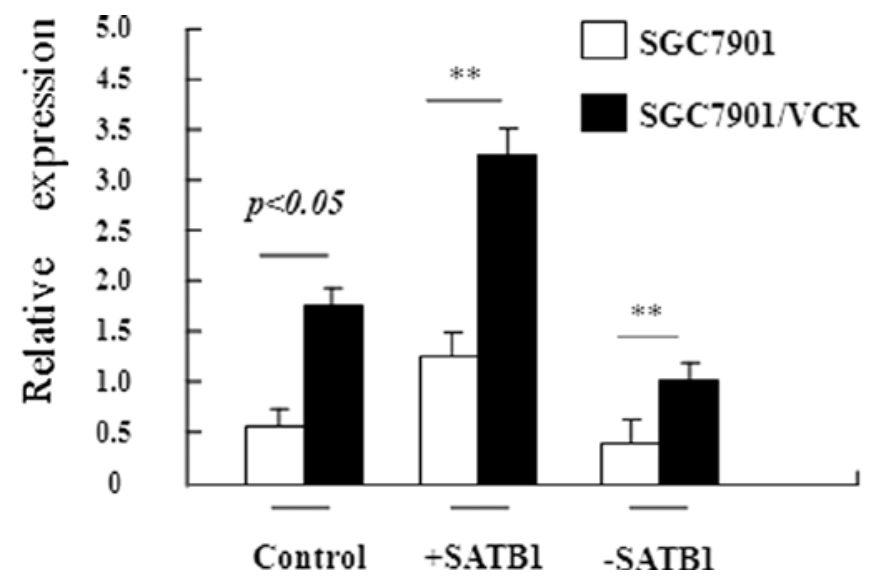

Figure 2. Relative expression level of SATB1 in SGC7901 cells and its variants. RT-PCR analysis revealed the relative SATB1 mRNA levels in different SGC7901-derived cell lines. GAPDH served as an internal control. The data are shown as the mean \pm SD from three independent experiments. ${ }^{* *} \mathrm{P}<0.05$, SGC7901 cells vs. SGC7901/VCR cells. The data are shown as the mean \pm SD from three independent experiments. +SATB1, cells transfected with SATB1 expression plasmid; -SATB1, cells transfected with SATB1-specific siRNA; SATB1, special AT-rich sequence binding protein 1; VCR, vincristine.

as expected in these stable cell lines (Fig. 2). The sensitivity of these cell lines to VCR was examined using the in vitro MTT assay (Fig. 3). Table I shows the $\mathrm{IC}_{50}$ values of these cells to VCR based on the MTT assay. Compared with SGC7901 
Table I. $\mathrm{IC}_{50}$ values indicating the sensitivity of the gastric cancer cell line SGC7901 and its variants to VCR.

\begin{tabular}{lc}
\hline Cell lines & $\mathrm{IC}_{50}$ value $(\mu \mathrm{g} / \mathrm{ml} ;$ mean $\pm \mathrm{SD})$ \\
\hline SGC7901 & $0.85 \pm 0.11$ \\
SGC7901-SATB1 & $1.53 \pm 0.45^{\mathrm{a}}$ \\
SGC7901-siRNASATB1 & $0.31 \pm 0.11$ \\
SGC7901/VCR & $3.15 \pm 0.37^{\mathrm{a}}$ \\
SGC7901/VCR-SATB1 & $4.66 \pm 0.38$ \\
SGC7901/VCR-siRNASATB1 & $1.61 \pm 0.15^{\mathrm{b}}$
\end{tabular}

$\mathrm{IC}_{50}$ values were calculated based on MTT assay and presented as the mean \pm SD of three independent experiments. ${ }^{\mathrm{P}} \mathrm{P}<0.01 \mathrm{vs}$. the control SGC7901 cells; ${ }^{b} \mathrm{P}<0.05$ vs. the control SGC7901/VCR cells. SATB1, special AT-rich sequence binding protein 1; VCR, vincristine.

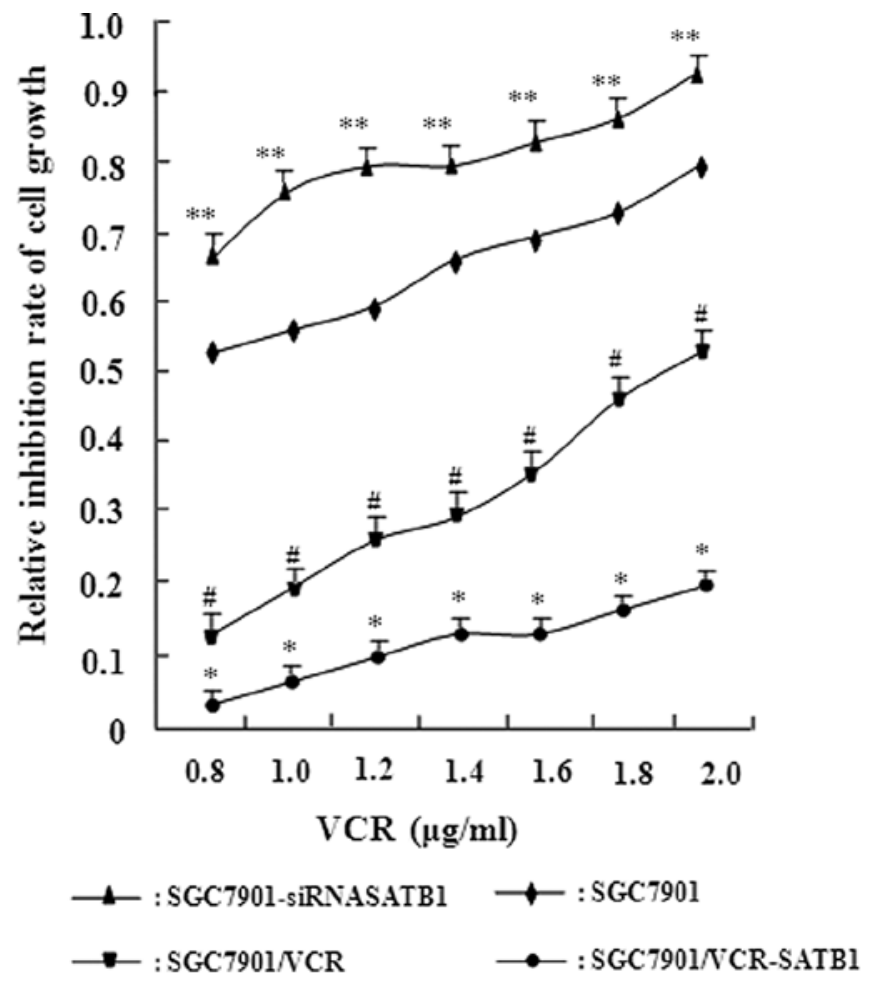

Figure 3. Positive correlation between the level of SATB1 expression and drug resistance in gastric cancer cells. MTT assay revealed the inhibition of cell proliferation by VCR in different SGC7901-derived cell lines. The relative inhibition rate was calculated using the formula described in Materials and methods. ${ }^{*} \mathrm{P}<0.01$ vs. SGC7901 cells; ${ }^{* *} \mathrm{P}<0.05$ vs. the control SGC7901 cells; ${ }^{*} \mathrm{P}<0.05$ vs. the control SGC7901/VCR cells. The data are shown as the mean \pm SD from three independent experiments. SATB1, special AT-rich sequence binding protein 1 ; VCR, vincristine.

cells, SGC7901-siRNASATB1 cells exhibited a significantly increased $\mathrm{IC}_{50}$ value for $\mathrm{VCR}(\mathrm{P}<0.05)$. A significant discrepancy in the $\mathrm{IC}_{50}$ value was also observed between SGC7901 and its MDR SGC7901/VCR cells $(\mathrm{P}<0.01)$. Moreover, a higher SATB1 expression in these cells was associated with a higher $\mathrm{IC}_{50}$ value. These results demonstrate the positive correlation between the level of SATB1 expression and drug resistance capacity in gastric cancer, suggesting that SATB 1 contributes to MDR in gastric cancer.
Table II. Intracellular VCR accumulation and retention in the gastric cancer cell line SGC7901 and its variants.

\begin{tabular}{llc}
\hline Cell lines & Accumulation & Retention \\
\hline SGC7901 & $74.48 \pm 5.54$ & $52.71 \pm 6.59$ \\
SGC7901-siRNASATB1 & $86.32 \pm 4.48^{\text {a }}$ & $33.75 \pm 3.41^{\text {a }}$ \\
SGC7901-SATB1 & $64.25 \pm 3.60$ & $33.75 \pm 3.41$ \\
SGC7901/VCR & $28.14 \pm 1.58^{\mathrm{c}}$ & $15.85 \pm 1.70^{\mathrm{c}}$ \\
SGC7901/VCR-SiRNASATB1 & $33.56 \pm 5.87$ & $18.97 \pm 1.20$ \\
SGC7901/VCR-SATB1 & $20.56 \pm 2.31^{\mathrm{b}}$ & $8.54 \pm 1.05^{\mathrm{b}}$
\end{tabular}

The values of VCR accumulation and retention were measured by flow cytometry and presented as the mean \pm SD of three independent experiments. ${ }^{\mathrm{a}} \mathrm{P}<0.05$ and ${ }^{\mathrm{b}} \mathrm{P}<0.05$ vs. their respective control cells. ${ }^{\mathrm{c}} \mathrm{P}<0.01$, SGC7901 vs. SGC7901/VCR cells. SATB1, special AT-rich sequence binding protein 1 ; VCR, vincristine.

SATB1 expression level is negatively correlated with intracellular VCR accumulation in gastric cancer cells. To explore the mechanism by which SATB1 contributes to MDR in gastric cancer, the effect of SATB 1 on intracellular VCR accumulation and retention was examined. Flow cytometry was performed to determine the fluorescence intensity of intracellular VCR in SGC7901 and its variant cell lines which expressed different levels of SATB1. The results showed that intracellular VCR accumulation in SGC7901/VCR cells was significantly less than that in the SGC7901 cells $(\mathrm{P}<0.01$, Table II). Moreover, the concentration of VCR in the SGC7901/VCR-SATB 1 cells was one-seventh of that in the SGC7901 cells, while the concentration of VCR was significantly increased in SGC7901-siRNASATB1 cells compared with SGC7901 cells $(\mathrm{P}<0.05)$. Taken together, these data show that the SATB1 expression level is negatively correlated with intracellular VCR accumulation in gastric cancer cells, which may explain how SATB1 contributes to MDR in gastric cancer.

SATB1 protects gastric cancer cells from VCR-induced apoptosis. It is widely accepted that protection against drug-induced apoptosis contributes to the development of MDR in cancer. Therefore, the effect of SATB1 on VCR-induced apoptosis was investigated in SGC7901 and SGC7901/VCR cells and their variants. Apoptotic cells were quantified as those that had a DNA content of $<2 \mathrm{~N}$ (sub-G $\mathrm{G}_{1}$ DNA content) (19). Results of the flow cytometric analysis revealed that SGC7901 cells had a higher apoptotic index than SGC7901/VCR cells $(\mathrm{P}<0.05)$. In addition, compared with SGC7901 and SGC7901/VCR cells, the apoptotic index was decreased in the cells which overexpressed SATB1 but increased in the cells in which SATB1 expression was depleted by siRNA $(\mathrm{P}<0.05$, Fig. 4$)$. These results indicate that SATB1 was able to inhibit the VCR-induced apoptosis of gastric cancer cells.

SATB1 promotes the invasion of gastric cancer cells. An in vitro invasion assay was performed to investigate the role of SATB1 in gastric cancer invasion. The results of this study revealed that the overexpression of SATB1 significantly promoted the migration of cells into the lower transwell chamber while 


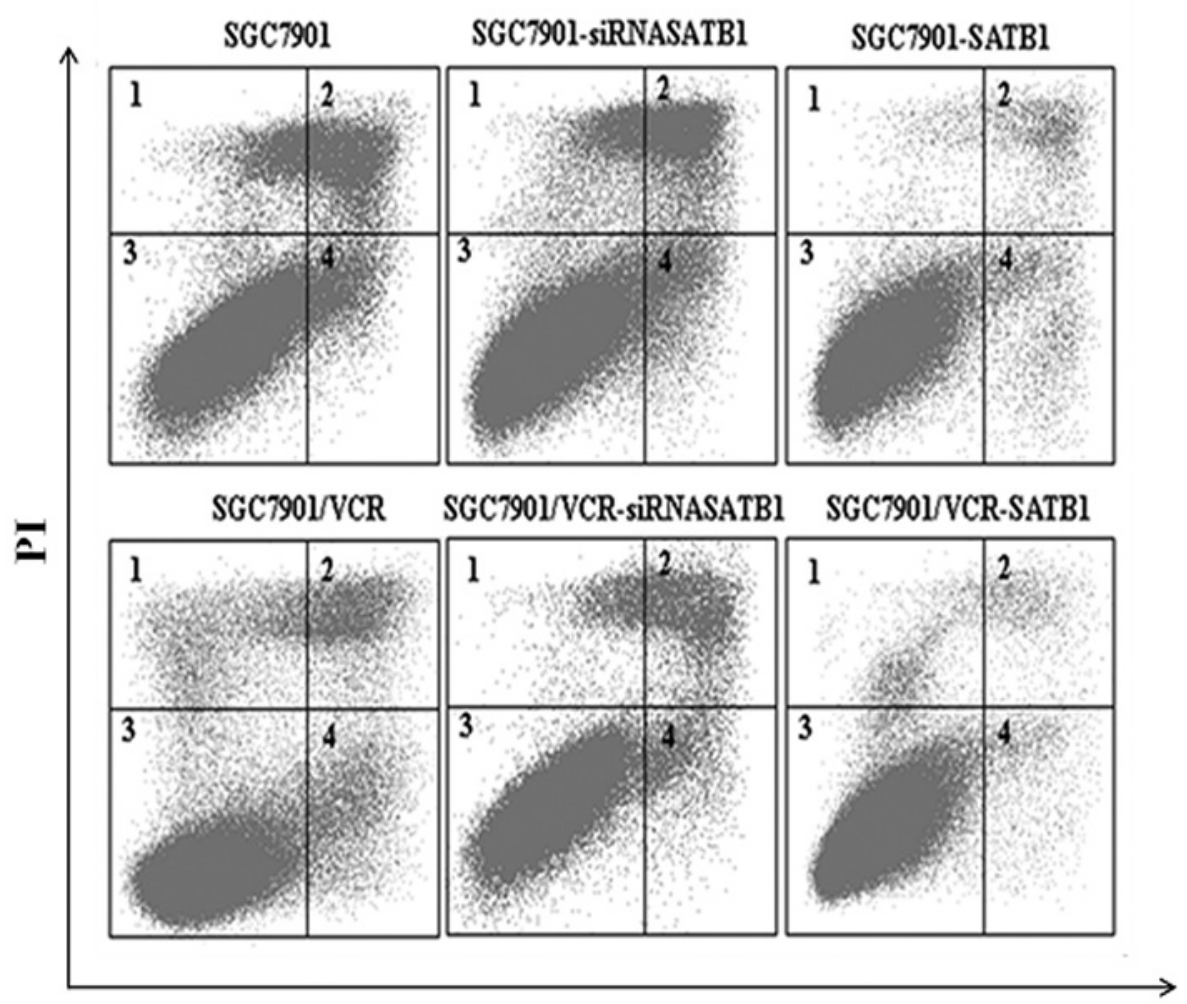

Annexin.V

Figure 4. SATB1 protects gastric cancer cells from VCR-induced apoptosis. The cells were incubated with $1 \mu \mathrm{g} / \mathrm{ml}$ VCR for $36 \mathrm{~h}$ and the apoptotic index was calculated based on flow cytometry and Annexin V/PI staining. The apoptotic index was 19.8, 35.0, 8.6, 10.6, 16.8 and 4.7\%, for SGC7901, SGC7901-siRNASATB1, SGC7901-SATB1, SGC7901/VCR, SGC7901/VCR-siRNASATB1 and SGC7901/VCR-SATB1 cells, respectively. SATB1, special AT-rich sequence binding protein 1; VCR, vincristine; PI, propidium iodide.

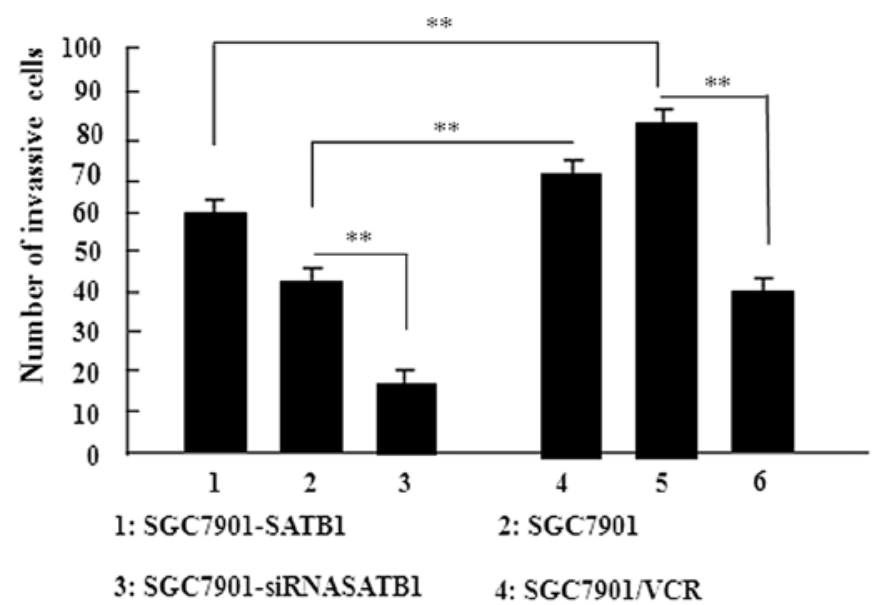

5:SGC7901/VCR-SATBl 6:SGC7901/VCR-siRNASAl

Figure 5. SATB1 promotes the invasion activity of gastric cancer cells. The invasion activity of the SGC7901 cell line and its derived cell lines was examined by in vitro transwell assay. Data are shown as the mean $\pm \mathrm{SD}$ of three independent experiments. ${ }^{* *} \mathrm{P}<0.05$ vs. the corresponding control cells. SATB1, special AT-rich sequence binding protein 1; VCR, vincristine.

siRNA-mediated depletion of SATB1 significantly inhibited cell migration into the lower chamber, compared with the control cells $(\mathrm{P}<0.05$, Fig. 5$)$. These data demonstrate that SATB1 promotes the invasion of gastric cancer cells.

\section{Discussion}

SATB1 was originally isolated as a nuclear factor that specifically binds to the BUR sequence (9). More recent studies have revealed that SATB1 is involved in the reconstruction of global genomes and regulates the expression of more than 1,000 genes. There is evidence to suggest that SATB1 modulates cell proliferation and lineage development and SATB1 is significant in breast cancer $(7,11,12,20)$. The mechanism by which SATB1 contributes to the malignant phenotypes of tumor cells is poorly understood.

MDR is known to be a significant aspect of malignant cancer and remains the major obstacle to the efficacy of cancer chemotherapy. Extensive research over the past decades has improved the understanding of the development of MDR in cancer cells. For example, over 60 genes have been identified as being upregulated in the drug-resistant human gastric cancer cell lines SGC7901/VCR and SGC7901/ADR (adriamycin) (21). However, no further studies have provided the mechanistic insight into the regulation of these genes.

Considering that SATB1 is a global regulator of gene expression, we speculate that the role of SATB1 is to regulate MDR in gastric cancer. The results of the present study support the role of SATB1 in MDR in gastric cancer. Western blotting and RT-PCR analysis were performed and we observed that SATB1 is overexpressed in VCR-resistant SGC7901 cells. Moreover, it was observed that the level of SATB1 expression was positively correlated with drug resistance in SGC7901 
cells. Collectively, these findings led to the conclusion that SATB1 contributes to the development of MDR in gastric cancer.

To explore the mechanism by which SATB1 contributes to MDR in gastric cancer, the effect of SATB1 on intracellular VCR accumulation and retention was examined. MDR has been investigated extensively for a number of years and a variety of mechanisms for MDR have been proposed, including increased drug efflux, decreased drug influx, DNA repair activation, detoxification and blockage of apoptosis. These mechanisms are not exclusive and may contribute to MDR in combination. Among them, the blockage of apoptosis and increased drug efflux are regarded as the most significant (22). Therefore, in this study, flow cytometry was performed to analyze the apoptotic rate in SGC7901-derived cells and it was observed that the expression level of SATB1 was negatively correlated with the apoptotic index in gastric cancer cells. Notably, VCR-induced apoptosis was largely eliminated by SATB1 overexpression in SGC7901 cells. These results are consistent with previous studies which revealed that SATB1 inhibited apoptosis in Sézary and breast cancer cells $(14,23)$, suggesting that the SATB1-mediated inhibition of apoptosis may contribute to MDR in gastric cancer.

Enhanced outward transport and reduced intracellular accumulation of anticancer drugs have been identified as major causes of MDR in certain tumors $(24,25)$. The present study revealed that VCR accumulation and retention occurred less frequently in SGC7901/VCR than in SGC7901 cells. Moreover, compared with the parental cells, VCR accumulation was inhibited in SATB1 overexpressing cells but was promoted in cells that were depleted of SATB1. A similar finding has been reported in breast cancer cells (25). ABC transporters usually function as pumps that remove toxins and drugs from the cells and p-glycoprotein (p-gp) and MDR-1 are the most characterized ABC transporters (26). Studies should therefore be conducted to investigate the effect of SATB1 on the expression of $\mathrm{p}$-gp and MDR-1, in order to elucidate whether SATB1 contributes to MDR by modulating the expression of $\mathrm{ABC}$ transporters and the intracellular accumulation of drugs.

Notably, a previous study revealed that the multidrug transporter $\mathrm{p}$-gp was involved in the migration and invasion of resistant melanoma cells, indicating a potential link between MDR and the invasive behavior of cancer cells (27). Therefore, the effect of SATB1 on the invasive behavior of gastric cancer cells was further investigated. An in vitro invasion assay demonstrated that gain or loss of SATB1 function was associated with the increased or decreased invasion activity of SGC7901 cells, providing complementary evidence that SATB1 promotes the invasion of gastric cancer cells. However, to provide further insight into the role of SATB1 in cancer invasion and metastasis, the role of SATB1 in modulating the expression of major genes involved in tumor invasion, including matrix metalloproteinases, requires examination.

In conclusion, the results of the present study reveal the upregulation of SATB1 in drug-resistant gastric cancer cells and that SATB1 contributes to MDR by inhibiting the accumulation of VCR in gastric cancer cells and protecting the cells from VCR-induced apoptosis. Addtionally, the significant role of SATB1 in the development of MDR in gastric cancer has been elucidated. SATB1 was also able to promote the invasion of gastric cancer cells. SATB1 may therefore be a promising target for the therapy of drug-resistant and invasive gastric cancer.

\section{Acknowledgements}

This study was supported by grants from the National Nature Science Foundation of China (no. 30901418 and no. 81172186). We would like to thank all members of our laboratory for their useful discussion.

\section{References}

1. Han HJ, Russo J, Kohwi Y and Kohwi-Shigematsu T: SATB1 reprogrammes gene expression to promote breast tumor growth and metastasis. Nature 452: 187-193, 2008.

2. Parker B and Sukumar S: Distant metastasis in breast cancer: molecular mechanisms and therapeutic targets. Cancer Biol Ther 2: 14-21, 2003

3. Hao ZH, Li X, Qiao T, Du R, Hong L and Fan D: CIAPIN1 congers multidrug resistance by upregulating the expression of MDR-1 and MRP-1 in gastric cancer cells. Cancer Biol Ther 5: 261-266, 2006.

4. Ozben T: Mechanisms and strategies to overcome multiple drug resistance in cancer. FEBS Lett 580: 2903-2909, 2006.

5. Pienta KJ and Smith DC: Advances in prostate cancer chemotherapy: a new era begins. CA Cancer J Clin 55: 300-318, 2005.

6. Spector DL: The dynamics of chromosome organization and gene regulation. Annu Rev Biochem 72: 573-608, 2003.

7. Cai S, Han HJ and Kohwi-Shigematsu T: Tissue-specific nuclear architecture and gene expression regulated by SATB1. Nature Genet 34: 42-51, 2003.

8. Yasui D, Miyano M, Cai S, Varga-Weisz P and KohwiShigematsu T: SATB1 targets chromatin remodelling to regulate genes over long distances. Nature 419: 641-645, 2002.

9. Dickinson LA, Joh T, Kohwi Y and Kohwi-Shigematsu T: A tissue-specific MAR/SAR DNA-binding protein with unusual binding site recognition. Cell 70: 631-645, 1992.

10. Agrelo R, Souabni A, Novatchkova M, Haslinger C, Leeb M, Komnenovic V, Kishimoto H, Gresh L, Kohwi-Shigematsu T, Kenner L and Wutz A: SATB1 defines the developmental context for gene silencing by Xist in lymphoma and embryonic cells. Dev Cell 16: 507-516, 2009.

11. Nakayama Y, Mian IS, Kohwi-Shigematsu T and Ogawa T: A nuclear targeting determinant for SATB1, a genome organizer in the T cell lineage. Cell Cycle 4: 1099-1106, 2005.

12. Alvarez JD, Yasui DH, Niida H, Joh T, Loh DY and KohwiShigematsu T: The MAR-binding protein SATB1 orchestrates temporal and spatial expression of multiple genes during T-cell development. Genes Dev 14: 521-535, 2000.

13. Zheng J: Is SATB1 a master regulator in breast cancer growth and metastasis? Womens Health (Lond Engl) 4: 329-332, 2008.

14. Li QQ, Chen ZQ, Xu JD, Cao XX, Chen Q, Liu XP and Xu ZD: Overexpression and involvement of special AT-rich sequence binding protein 1 in multidrug resistance in human breast carcinoma cells. Cancer Sci 101: 80-86, 2010.

15. Lu X, Cheng C, Zhu S, Yang Y, Zheng L, Wang G, Shu X, Wu K, Liu K and Tong Q: SATB1 is an independent prognostic marker for gastric cancer in a Chinese population. Oncol Rep 24: 981-987, 2010.

16. Cheng GZ, Chan J, Wang Q, Zhang W, Sun CD and Wang LH: Twist transcriptionally up-regulates AKT2 in breast cancer cells leading to increased migration, invasion, and resistance to paclitaxel. Cancer Res 67: 1979-1987, 2007.

17. Li QQ, Wang WJ, Xu JD, Cao XX, Chen Q, Yang JM and Xu ZD: Involvement of CD147 in regulation of multidrug resistance to P-gp substrate drugs and in vitro invasion in breast cancer cells. Cancer Sci 98: 1064-1069, 2007.

18. Shi Y, Zhai H, Wang X, Han Z, Liu C, Lan M, Du J, Guo C, Zhang Y, Wu K and Fan D: Ribosomal proteins S13 and L23 promote multidrug resistance in gastric cancer cells by suppressing drug-induced apoptosis. Exp Cell Res 296: 337-346, 2004.

19. Maxwell SA and Davis GE: Biological and molecular characterization of an ECV-304-derived cell line resistant to p53-mediated apoptosis. Apoptosis 5: 277-290, 2000. 
20. Wen J, Huang S, Rogers H, Dickinson LA, Kohwi-Shigematsu T and Noguchi CT: SATB1 family protein expressed during early erythroid differentiation modifies globin gene expression. Blood 105: 3330-3339, 2005.

21. Zhao Y, You H, Liu F, An H, Shi Y, Yu Q and Fan D: Differentially expressed gene profiles between multidrug resistant gastric adenocarcinoma cells and their parental cells. Cancer Lett 185 211-218, 2002.

22. Gottesman MM, Fojo T and Bates SE: Multidrug resistance in cancer: role of ATP-dependent transporters. Nat Rev Cancer 2: 48-58, 2002.

23. Wang Y, Su M, Zhou LL, Tu P, Zhang X, Jiang X and Zhou Y: Deficiency of SATB1 expression in Sezary cells causes apoptosis resistance by regulating FasL/CD95L transcription. Blood 117: 3826-3835, 2011.
24. Gorlick R and Bertino JR: Drug resistance in colon cancer. Semin Oncol 26: 606-611, 1999.

25. Naito S, Yokomizo A and Koga H: Mechanisms of drug resistance in chemotherapy for urogenital carcinoma. Int J Urol 6: 427-439, 1999.

26. Dong $X$ and Mumper RJ: Nanomedicinal strategies to treat multidrug-resistant tumors current progress. Nanomedicine (Lond) 5: 597-615, 2010.

27. Colone M, Calcabrini A, Toccacieli L, Bozzuto G, Stringaro A, Gentile M, Cianfriglia M, Ciervo A, Caraglia M, Budillon A, et al: The multidrug transporter P-glycoprotein: a mediator of melanoma invasion? J Invest Dermatol 128: 951-971, 2008. 